\title{
Hybrid Simulation for System-Level Structural Response
}

\author{
Justin Adam Murray ${ }^{1}$, Mehrdad Sasani ${ }^{2}$, and Xiaoyun Shao ${ }^{3}$
}

Abstract

Hybrid simulations combine physical and analytical components into a single simulation

5 toevaluate the response of a structure, often under seismic ground motion. This allows an experiment to be conducted in which structural components with complex response can

7 bemodeled experimentally and more well-known componentscan be represented within an analytical model. The coordination software UI-SimCor, developed by the MUST-SIM NEES

9 facilityat the University of Illinois at Urbana-Champaign, is a hybrid simulation tool which

10 performs the dynamic analysis and other software and hardware coordination tasks for hybrid

11 simulations. In many hybrid simulations, including those that have used UI-SimCor, analytical

12 models with few effective degrees of freedom are typically used. In simulations where system-

13 level behaviors and the response of the analytical components are of importance, a more detailed

14 analytical system is needed. This changeover to a more complex analytical system and increase

15 in general complexity of the hybrid simulation can cause various issues within the UI-SimCor

16 framework. This study discusses the difficulties and issues that arise from having large and

17 complex analytical substructures in hybrid simulation, and the effective mitigation or solutions to

18 those problems.

19 Keywords: Hybrid Simulation, Reinforced Concrete, Large-Scale Testing, OpenSees, Structural 20 Failure

\footnotetext{
${ }^{1}$ PhD Candidate, Department of Civil and Environmental Engineering, Northeastern University, Boston, MA, Email: murray.jus@husky.neu.edu

${ }^{2}$ Associate Professor, Department of Civil and Environmental Engineering, Northeastern University, Boston, MAEmail:sasani@neu.edu, Tel: 617-373-5222,Fax: 617-373-2444

${ }^{3}$ Associate Professor, Department of Civil and Construction Engineering, Western Michigan University, Kalamazoo, MI, Email: xiaoyun.shao@wmich.edu 


\section{Introduction}

Hybrid simulations (HS) combine physical and analytical components (substructures)

23 into a single simulation toobtain a system-level structural response under dynamic ground

24 motion (GM). HS allows forevaluation ofentirebuilding systems, with the potential for

25 experimental testing of specific structural components at large scale so thatboth experimental and

26 analytical behaviors and theinteraction between them can be observed. One common practice

27 ofHS is that complex mechanisms or componentsare modeled experimentally (and often at large-

28 scale) while more well-known componentsare represented within an analytical model.

29 This study focuses on the development of a HS approach to help achieve the final goal of

30 a Network for Earthquake Engineering Simulation Research (NEESR) project, which is to

31 evaluate system level response of existing reinforced concrete (RC) structures following shear-

32 axial column failure. Although the findings and observations presented herein are applicable in

33 manyHSprocedures, this study and the presented advancements are related to the use of the UI-

34 SimCor coordination software at the University of Illinois (Kwon et al., 2005, Kwon et al., 2008).

35 Other similar architectures for HS would similarly benefit from the advancements presented in 36 this study.

37 Shear-axial column failure, and the system-level collapse resistance of a building after 38 such a failure, is an important topic in RC retrofitting and seismic analysis, as many buildings

39 built prior to the mid-1970s have column detailing which make them susceptible to shear-axial 40 failure. Shear-axial column failure is a difficult mechanism to predict, and current failure models 41 and capacity equations include a high degree of uncertainty and variability (Lynn et al., 1996; 42 Elwood, 2004;Sasani 2007; Henkhaus et al., 2013). However, the continuous development of HS 
43 makes it feasible to analyze a building'sresponse to such a column failureandto understand the

44 collapse-resisting mechanisms of the structure under such extreme conditions.

45 Modern HS originated from the pseudodynamic testing method firstly reported by Takanashi

46 et al. (1975). Further research on the use of the pseudodynamic method for seismic testing, such

47 as quantifying experimental errors, limitations, and stability issues, was conducted by Mahin and

48 Shing (1985). HS methods have since underwent an accelerated development phase reflected by

49 increasingly complex analytical and physical substructures, employment of high-performance

50 hydraulic loading equipment and development of various HS coordination software (Elnashai et

51 al. 2004, Shing et al, 2007). HS have also been conducted with geographic distribution of test

52 hardware and software. Yang et al. (2009) performed a geographically distributed HS, testing

53 zipper frames with components of the test located at both the University of California at

54 Berkeley and at University of Colorado at Boulder. The displacement-based pseudodynamic

55 HSmethod prevails in recent implementations (Shao and Griffith, 2013) due to its similar testing

56 setup to a quasi-static test and the many available supporting open-source programs, such as

57 OpenFresco (Schellenberg et al., 2007) and UI-SimCor (Kwon et al., 2005, Kwon et al., 2008).

58 Hashemiet al. (2014) discuss the challenges regarding convergence and spurious oscillation

59 that can occur when conducting HS with large numerical substructures. One way to address the

60 challenge of complex numerical modeling in HS is to develop HS coordinating systems that

61 utilize the dynamic solution algorithms within existing FEM software. For example Wang et al.

62 (2008) developed a P2P HS system that combines physical testing with numerical substructures

63 modeled in OpenSees and ABAQUS. The architecture of the P2P system allows the equations of

64 motion to be formulated and solved within each substructure's domainusing their respective

65 software and only maintaining the displacement and force balances at the boundaries using an 
66 initiative algorithm. Another example is OpenFresco (Schellenberg et al., 2010) which serves as

67 a middleware between physical testing and a numerical simulation conducted in OpenSees. Thus

68 OpenSees is utilized not only for numerical modeling, but also for the dynamic solution of the

69 entire structure model being investigated. Further advancement in OpenFrescowere made

70 recently byHashemi et al. (2014) who proposed an innovative overlapping substructuring

71 technique for collapse HS of steel frame when large deformations were anticipated.

72 On the other hand, UI-SimCor adopted a different HS coordinator architecture, and is the

73 primary coordinator used at the MUST-SIM lab atthe University of Illinois at Urbana-

74 Champaign (UIUC).So far UI-SimCor has been successfully implemented in large scale HS of

75 RC bridgesof various configurations (Spencer et al. 2006, Kim et al., 2011, Abdelnaby et al.,

76 2014) and semi-rigid steel frames (Mahmoud and Elnashai, 2013). It has also been used for small

77 scale HS of 6-story steel frames (Lin, 2010) and RC bridges (Li et al., 2013). While some of

78 these experiments have complex numerical substructures, they had a limited number ofeffective

79 DOFs (discussed in the following section).

In this study, a 10-story RC building is analyzed under a severe earthquake GM, and is

81 substructured for HS to allow physical experimentation of three lower-floor columns in the

82 MUST-SIM NEES facility at UIUC, with the remainder of the building system modeled in the

83 computer program OpenSees (McKenna et al., 2002). A large three-dimensional analytical

84 substructure (thousands of DOFs)is used with nonlinear modeling of all elements, as collapse-

85 resistance mechanisms in building structures often involve large amounts of inelastic

86 deformation. The challenges that arise from using such a complex numerical model and potential

87 adjustments to the HS coordinator/architecture in order to mitigate these challenges is presented

88 in the following sections. 
90 In this section, the analytical model that is used in the HS is presented. Note again that in

91 this paper the main objective is to develop a HS method that is applicable for evaluating the

92 response of such a model and to demonstrate the issues and the proposed solutions. To conduct a

93 successful HS, which will allow for evaluation ofsystem-level behaviors of older RC buildings

94 subjected to seismic GM, a representative pre-1970s 10-story RC building structure is designed

95 according to ACI 318-63 (1963), and a detailed analytical model of the structure is developed.

96 The building is designed based on a review of the design and layout of several actual RC

97 structures constructed during that time period (John A. Blume \& Associates, 1973). Typical

98 building characteristics included tall $1^{\text {st }}$ stories (for lobbies, etc.), as well as deep spandrel beams

99 with shallower interior beams, allowing for more interior space and higher ceilings, while putting

100 more of the seismic demand on the exterior of the structure. More discussion of the review of

101 pre-1970s construction can be found in Murray and Sasani (2013). For the representative

102 structure, the building plan consists of two 6.10m (20') spans in the north-south (transverse)

103 direction and six 6.10m(20') spans in the east-west (longitudinal) direction. The structure has

$104400 \mathrm{mmx} 710 \mathrm{~mm}$ (16"x28”) spandrel beams on the first floor with 400mmx560mm (16"x22”)

105 spandrel beams on other floors. Interior beams are 400mmx460mm (16"x18") on all floors. The

106 first story height is $4.27 \mathrm{~m}\left(14^{\prime}-0\right.$ '), while other stories have a height of $3.20 \mathrm{~m}$ (10'-6”). Floors

107 consist of one-way $115 \mathrm{~mm}(4.5$ ”) slab, supported by intermediate beams at the midpoint of

108 longitudinal beams. Concrete is designed with an f'c=27.6 MPa (4 ksi) and all steel is Grade 60.

109 A typical floor plan is shown in Figure 1. Note that the second floor is identified as the top of the

110 first story. 
112 modeling software capable of nonlinear modeling of building structures. Two-node beam-

113 columnelements are used to model all beams, columns, and slabs within the structure. Elements

114 are nonlinear using a concrete constitutive relationship with no tensile strength, and a bilinear

115 steel model with $2 \%$ strain hardening. A force-based formulation is used for the beam elements,

116 which compared to a displacement formulation, allows for a reduced number of nodes and

117 elements in the model. Columns of the structure have 4 integration points while beams and slabs

118 have 3 integration points (but are represented with more elements).The slabs of the floor system

119 are not modeled as shells, but rather as a grid of nonlinear beam elements (sized based on their

120 tributary areas), using half of their linear torsional stiffness(MacLeod, 1990). Nonlinear shell

121 elements are not used because they have not been extensively verified in OpenSees, and the use

122 of a grid of beam elements representing slabs has been successfully used in the past with good

123 agreement with physical data (e.g. Sasani et al., 2011). Beam sections are modeled as T and L

124 sections to account for the contribution of the adjacent slab. The layout of nodes and connecting

125 elements in the structural model in OpenSees are shown for a typical floor plan in Figure 2. Note

126 that the node clusters around columns are used for modeling the rigid joints of the system and the

127 bar-slip deformations at the faces of the joints.Joint damage is outside the scope of this study so

128 the joints are modeled using stiff linear elements. Bar-slip deformations were accounted for

129 through modification of the Gauss-Lobatto integration point weights to amplify deformations at

130 the element ends. The amount of amplification was calibrated against column tests by Sezen

131 (2002) and Lynn et al. (1996).

132 The gravity loads of the structure are distributed among all of the nodes in the floor based on 133 their tributary area. The live load is assumed to be $3.11 \mathrm{kN} / \mathrm{m}^{2}$ (65psf) for design, which is used 
134 as an average value representing the live load applied to different parts of the floors. The floors

135 are also subjected to $1.92 \mathrm{kN} / \mathrm{m}^{2}$ (40psf) of dead load in addition to the weight of the structural

136 components, accounting for partitions, floor finishing, drop ceilings, as well as mechanical,

137 piping and fixtures. Masses used in the seismic analysis are distributed among all of the nodes,

138 similar to the distribution of the gravity forces, and include the dead load and $25 \%$ of the live

139 load (GSA, 2003). Bar-slip behavior is explicitly accounted for in the OpenSees model. Bar-slip

140 is a flexural response that occurs in $\mathrm{RC}$ elements which results in concentrated rotations at the

141 end of beams and columns due to strain penetration of the rebar into joints and foundations. The

142 model used in this study was calibrated against several large-scale tests which measured the

143 effect of these rotations on the response (Lynn et al., 1996; Sezen, 2002). As the purpose of this

144 paper is to discuss the HS process as it relates to a large analytical model and not the specifics of 145 modeling techniques in OpenSees, further discussion of the details of the analytical model are 146 not presented here. Formore info regarding analytical modeling techniques used in this project, 147 see (Murray and Sasani 2013 and 2014).

\section{Overview of Statically-Condensed Hybrid Simulation (SC-HS)}

149 Within pseudodynamic HS, there are several methods used to analyze the structural system as 150 a whole, generally through a centralized coordinator. Coordinators typically handle various tasks

151 within a HS, including communication between substructures, solution of the equation of motion, 152 and data logging. OpenFresco and UI-SimCor remain the most popular tools for coordinating a 153 HS in the US.

154 A static condensation can be used to eliminate from dynamic analysis those degrees of 155 freedom (DOFs) of a structure to which zero mass is assigned (Chopra, 2012). In a HS due to the 
nature of the procedure, one usually includes not only DOFs that have mass, but also the ones

157 which have gravity (or other static) loads applied as well as the DOFs at the boundaries between

158 different substructures. These DOFs are collectively called "effective" DOFs in this paper. HS

159 using such static condensation will from this point forward is defined as a statically-condensed

160 hybrid simulation (SC-HS). SC-HS can be described using the following partitioned differential

161 equation, which represents the equilibrium of all DOFs of the structure.

162

$$
\left[\begin{array}{cc}
m_{e} & 0 \\
0 & 0
\end{array}\right]\left\{\begin{array}{l}
\ddot{u}_{e} \\
\ddot{u}_{s}
\end{array}\right\}+\left[\begin{array}{cc}
k_{e e} & k_{e s} \\
k_{e s} T & k_{s s}
\end{array}\right]\left\{\begin{array}{l}
u_{e} \\
u_{s}
\end{array}\right\}=\left\{\begin{array}{l}
P_{e} \\
P_{s}
\end{array}\right\}
$$

163 where $\ddot{u}$, and $u$ are the acceleration and displacement vectors of the system. For clarity of the

164 discussion that follows, the damping term(a function of the velocity vector $\dot{u}$ ) is excluded

165 fromthe equation and will be discussed later. The subscript $e$ represents the effective DOFs

166 whose response will be evaluated solvingthe dynamic equations of motion. The subscript $s$

167 represents the remaining DOFs of the structure, whose response will be evaluated byeither

168 solving static equilibrium equations or experimentally. $m_{e}$ is the lumped mass matrix associated

169 with the effective DOFs, which has some zero diagonal terms associated with the DOFs which

170 either are at the boundaries of the substructures or have gravity loads, but no mass. The $\mathrm{k}$

171 submatrices in the second term in (1) represent the portions of the stiffness matrix of the

172 structure associated with the effective and the remaining DOFs as well as the corresponding

173 cross terms.Based on the definition of the effective DOFs presented in the previous paragraph,all

174 the components of the $P_{S}$ vector are zero. This is due to the fact that $P_{e}$ includes all static and

175 dynamic forces applied to the structure. But for a more general definition of effective DOFs,

176 which will be discussed later, $P_{S}$ is not replaced with a zero vector yet.

177 The partitioned equations in (1) are 


$$
m_{e} \ddot{u}_{e}+k_{e e} u_{e}+k_{e s} u_{s}=P_{e}
$$

$$
k_{s s} u_{s}=P_{s}-k_{e s}{ }^{T} u_{e}
$$

180 From (2b) $u_{s}$ can be found as

$$
u_{s}=\left(k_{s S}\right)^{-1} P_{s}-\left(k_{s s}\right)^{-1} k_{e s}{ }^{T} u_{e}
$$

and replacing $u_{s}$ from (3) in (2a), we arrive at

$$
m_{e} \ddot{u}_{e}+k_{e e}^{*} u_{e}=P_{e}-k_{e s}\left(k_{s s}\right)^{-1} P_{s}
$$

184 where $k_{e e}^{*}$ is the statically condensed stiffness matrix of the structure.

$$
k_{e e}^{*}=k_{e e}+k_{e s}\left(k_{s s}\right)^{-1} k_{e s}^{T}
$$

In (4a) if $P_{S}$ is set equal to zero $\left(P_{S} \neq 0\right.$ will be discussed later $)$ we will have

$$
m_{e} \ddot{u}_{e}+k_{e e}^{*} u_{e}=P_{e}
$$
linear combination of $m_{e}$ and $k_{e e}^{*}$.

$$
m_{e} \ddot{u}_{e}+c_{e e}^{*} \dot{u}_{e}+k_{e e}^{*} u_{e}=P_{e}
$$

In a linear structure, $u_{e}$ and then $u_{s}$ can be found from (6) and (3), respectively. Under a 192 general loading condition and particularly for a nonlinear structure,(6) is solved incrementally.

$$
m_{e} \Delta \ddot{u}_{e}+c_{e e}^{*} \Delta \dot{u}_{e}+k_{e e}^{*} \Delta u_{e}=\Delta P_{e}
$$

The dynamic analysis in SC-HS can be conducted using a non-iterative time-integration

195 scheme. The operator splitting method in conjunction with the $\alpha$-modified Newmark scheme $(\alpha-$ 196 OS method) has been implemented as the integration algorithm in UI-SimCor. The $\alpha$-OS 
197 integration scheme was proposed by Combescure and Pegon (1997). Compared to an iterative

198 implicit integration scheme for pure numerical simulation, the $\alpha$-OS method does not require

199 iterations within each time step to achieve equilibrium for nonlinear structural responses, which

200 is a desired feature for HS purposes, as perturbating or iterative motion of a physical specimen

201 can be avoided.The $\alpha$-OS method uses the initial stiffness of all substructures to solve (7), that is,

202 it replaces $k_{e e}^{*}$ with the statically condensed initial stiffness $k_{I}$.

203 Due to the nonlinearity of the system, the solution to (7) needs to be corrected. For this 204 purpose, the solution (incremental displacements at effective DOFs) is sent as commands to be 205 executed by each analytical and experimental substructure toobtain the resisting forces. To 206 evaluate the status of total equilibrium in nonlinear systems, $k_{e e}^{*} u_{e}$ in (6) must be replaced by the 207 resisting force vector $p_{r}^{*}\left(u_{e}\right)$

$$
m_{e} \ddot{u}_{e}+c_{e e}^{*} \dot{u}_{e}+p_{r}^{*}\left(u_{e}\right)=P_{e}
$$

209 Within a SC-HS, all substructures, regardless of their size or whether they are experimental or 210 analytical, are treated equally and are collectively referred to as substructures or 'modules'. The 211 experimental substructures find their resisting force in the laboratory. The analytical substructure 212 of a HS would need to apply the displacement commands through some form of constraint 213 problem. A flow chart showing the sequence of steps in a SC-HS performed in UI-SimCor is 214 shown in Figure 3, as it pertains to this project. During initialization, information regarding the 215 model and analysis, such as the mass, damping, and initial stiffness matrices, are defined. 216 Stiffness matrices are defined for each module individually and assembled into a global stiffness 217 matrix by the program. 
218 Note that as shown in Figure 3, simulation coordinators handle several aspects of a SC-HS.

219 They perform the dynamic time-integration solved under the defined loading or GM. They also

220 typically handle the communication between various substructures of the simulation, passing

221 displacements commands onto physical specimens and analytical models as well as collecting

222 the restoring forces and assembling the restoring force vector. Some coordinators, such as UI-

223 SimCor, have additional capabilities in terms of graphical user interfaces for tracking data in real

224 time, triggering data acquisition (DAQ) systems, as well as automatic control of cameras in the

225 laboratory.

226 SC-HS has typically been used to analyze systems with few effective DOFs to minimize

227 computational effort within the coordinator, since the coordination software solves both static

228 (for gravity) and dynamic equilibrium equations, in addition to performing various other tasks.

229 Sometimes the complexity amounts to as little as 1 or 2 effective DOFs. Note that this does not

230 inherently limit the complexity of the analytical modules, which can have orders of magnitude

231 more DOFs than the model analyzed by the coordinator, and are solved within a structural

232 analysis software. Due to the required distribution of masses and loads (for near-collapse

233 analysis of a 3D structure) in this study, was a need to drastically increase the number of

234 effective degrees of freedom in the HS beyond what has been done in UI-SimCor in the past.

235 Note again that other tests have included many DOFs, but typically have had few effective DOFs

236 (masses, loads, boundary locations). This paper will discuss the process and difficulties of doing 237 so. 


\section{Issues with SC-HS for Large Analytical Systems}

239

240 Previous HSs have noted that the higher-mode response of a system can be erroneously

241 excited causing spurious oscillations between time steps (Nakashima and Kato 1988;

242 Combescure and Pegon, 1997). Combescure and Pegon proposed the use of alpha-factor

243 numerical damping (alpha-OS method) to dissipate these higher-mode contributions from the

244 results. However, in our analyses of the 10-story RC structure with a large number of effective

245 DOFs, the alpha-damping did not mitigate the extreme oscillations that can occur in the 246 simulation.

247 To illustrate the oscillations that can occur, an analytical example was conducted, in which 248 the 10-story system is represented by an OpenSees model acting as the only module 249 (substructure) communicating with UI-SimCor. There are a total of 470 effective DOFs in the 250 model (470 masses and static loading points). The analysis consists of 100 steps of gravity 251 loading, followed by 100 steps of stabilization, in which no additional loads are applied. Shortly 252 after the start of the dynamic portion of the analysis (Step 200), the large oscillations of higher253 mode frequencies begin to occur. Figure 4 shows the axial force in exterior first story column B1. 254 This axial force shows an oscillation comparable to the magnitude of the gravity load itself. 255 Similar behavior can be found in other localized DOFs. Note that although there are several 256 thousand total DOFs in the structural model in OpenSees, the equation of motion solved by UI257 SimCor is solved on a condensed system of 470 DOFs, so the massless DOFs are not a factor in 258 the cause of these oscillations. 
Note that an alpha factor of 0.05 was used, which is the suggested value based on the UI-

260 SimCor manual (Kwon et al., 2007). This should provide moderate amounts of numerical

261 damping. The alpha factor can range from 0 to $1 / 3$, providing various amounts of numerical

262 damping. Also, the Rayleigh damping is defined with a stiffness proportional component, so the

263 model is significantly overdamped in the high-frequency modes (damping was set to $5 \%$ in the

$2641^{\text {st }}$ and $2^{\text {nd }}$ modes in transverse direction). Even with these damping parameters in place the

265 oscillations are unable to dissipate. If only mass-proportional damping was used, the oscillation

266 would become more significant, since mass-proportional damping results in low damping in

267 short-period (higher-frequency) modes. Therefore, although this study suggests the use of

268 Rayleigh damping in conjunction with the alpha-OS method, it is clear that other mitigation

269 techniques are needed to reduce the likelihood of spurious oscillations in the model results. Note

270 that these oscillations occur even in purely analytical systems, and are therefore not related in

271 any way to experimental errors.

\section{Convergence and static solution of analytical modules}

273 One of the most difficult issues when analyzing large structural systems using SC-HS is that

274 all modules(substructures) are treated equally, with the central coordinatorsending displacement

275 commands to be solved statically by each substructure. When one of the substructures contains a

276 large number of effective DOFs, this creates a large and potentially demanding constraint

277 problem which is difficult to solve statically. This can lead to convergence issues when the

278 system is modeled with various nonlinearities. As a result, hybrid analyses with a large number

279 of effective DOFs (masses and loads) can exhibit issues both with high-frequency oscillation, as

280 described above, and with convergence due to the difficult solution of a large constraint problem. 
281 Other hybrid simulations have also shown stability issues with explicit time integration in

282 different architectures using OpenFresco as the coordinator (Del Carpio et al., 2015).

\section{Solutions for using existing SC-HS frameworks for large models}

284 294 problem solved statically in a large numerical substructure can easily cause issues. Therefore in 295 SC-HS, it is more important to consolidate masses and gravity loadsto reduce the number of 296 effective DOFs solved in the equation of motion rather than to remove nodes and elements from 297 the analytical model representing one of the substructures. This addresses multiple issues:

\section{Reducing model DOFs through consolidation and symmetry}

To address the problems associated with the use of large models in SC-HS, several mitigation techniques are used in parallel. Since the primary issues stem from the size of the model, the most basic and effective technique for alleviating these issues is by simplifying the analytical model to the extent possible. Note that the techniques described below are primarily meant to resolve issues in SC-HS architectures, such as UI-SimCor, and are less applicable to other methods such as the use of OpenFresco.This is because the primary issues arise in the static solution of the large substructure, not the dynamic alpha-OS solution. The unconditional stability of the alpha-OS method (provided that the stiffness matrix in the formulation is larger than the actual stiffness) dictates that few issues arise in the dynamic solution. Rather, the constraint

- It cuts down on the amount of communication passed back and forth between the substructures and the coordinator;

- It reduces the number of constraints being applied to the building module, reducing the likelihood of convergence issues; 
- With a more coarse distribution of mass, the number of vibration modes (and hence the highest frequencies in the system) becomes lower, somewhat reducing the likelihood or impact of spurious oscillations during the time integration.

305 However, depending on the purpose of the HS and the reliance on accurate or detailed 306 analytical modeling, there is a limit to how simplified of a model is acceptable. Additionally,

307 although it is often acceptable to lump masses more coarsely in a lateral seismic analysis of a 308 building structure, if such an analysis also aims to achieve reasonable gravity forces, the vertical 309 loads of the system should still be assigned with a more detailed distribution (for correct beam 310 moments, etc.). Since vertical loads are also treated as effective DOFs, they represent a portion 311 of the model complexity which is difficult to reduce or simplify without severely sacrificing the 312 validity of the gravity loading.

313 Since reduction of model complexity is shown to be one of the most effective ways of 314 achieving better and more stable results, it is also advisable to take advantage of symmetry of the 315 structure and its loadings whenever possible. For instance, since the representative structure is 316 symmetric about line 4 in Figure 1, and the HS consists of ground motion only in the N-S

317 direction, the model can effectively be cut in half, significantly reducing the number of DOFs.

318 New gravity application for reduction of effective DOFs

319 In the HS described previously, any DOFs that had gravity loads needed to be included in the 320 effective DOFs. A method is presented here to apply the gravity loading to DOFs that need only 321 exist outside of the coordination software (do not need to be included in the dynamic analysis 322 involving effective DOFs), allowing for the removal of any effective DOFs purely associated 323 with gravity loads. This means that the static loads are moved from $P_{e}$ to $P_{s}$, and in (1) to 
$324(4) P_{s} \neq 0$.Following this modification, the effective DOFs include only the DOFs that have mass 325 or are at the boundaries of the substructures.

326 The gravity analysis is conducted in two steps. In the first step, the loads are applied within 327 the analytical substructure prior to UI-SimCor being initialized with the modules. In this step, all 328 the effective DOFs are constrained from motion (i.e. $u_{e}=0$ ). If the structure were linear, the 329 solution could be found using (3): $u_{s}=\left(k_{s s}\right)^{-1} P_{s}$ (note that $k_{s S}$ is the stiffness matrix of the 330 structure under the condition that $u_{e}=0$ ). In the nonlinear structure studied here, this analysis is 331 conducted iteratively within OpenSees.

332 Then the modules are initialized within UI-SimCor and the second step of the gravity analysis 333 is performed using a static analysis in SimCor. Obviously, the gravity load vector defined in UI334 SimCor is set to zero. UI-SimCor will then resolve the unbalanced forces at the effective DOFs, 335 balancing the load incrementally between the substructures. This method proved to work well, 336 with similar success to the original gravity load method implemented in UI-SimCor, but with 337 reduced effective DOFs.

338 The method described above can have varying levels of efficiency in regards to the reduction 339 of effective DOFs, largely related to the ratio of discrete gravity loading locations to DOFs with 340 mass. For instance in an extreme case, in a building model such as the one used in this study, a 341 typical floor can be composed of 275 nodes. If gravity is distributed throughout all of those 342 nodes based on their tributary areas, but the dynamic analysis is conducted only laterally with 343 rigid diaphragm action, then the effective DOFs in UI-SimCor can be reduced from 276 per floor 344 (275 vertical gravity DOFs and 1 lateral mass at the center of the structure) down to 1 (just the 345 lateral mass), removing $99.6 \%$ of the DOFs from the equation of motion.In the case of our 346 structural model and distribution of masses in this system, vertical and lateral masses were 
347 lumped at the column nodes (12 nodes, 24 masses per floor), so this resulted in a $91.6 \%$

348 reduction in the \# of DOFs in the equation of motion for this study.

\section{Reducing oscillating through damping and time step size reduction}

350 The above methods are all used in this project to reduce the number of masses and effective

351 DOFs located within the coordination software for SC-HS. Additionally, different forms of

352 damping can be used in an attempt to further decrease the likelihood of spurious oscillations of

353 higher mode behaviors. As discussed earlier, increasing the alpha factor in the alpha-OS method

354 can introduce higher amounts of numerical damping which has been shown to mitigate

355 oscillations in analysis results (Combescure and Pegon, 1997). Through a series of analyses on

356 systems that were showing oscillation, an alpha factor of 0.1 was found to be most effective at

357 reducing that oscillationin this study. Earlier it was discussedthat problems (oscillations) can

358 arise associated with mass proportional damping with UI-SimCor analyses due to the low

359 dissipation of high-mode behavior. The stiffness proportional term of the Rayleigh damping

360 causes asymptotically increasing damping ratios as the period of vibration approaches zero. This

361 can help dissipate vibrations that occur in localized modes of the system, which tend to be the

362 problematic areas.

363 Another solution to the oscillations that can occur in hybrid analyses, is to reduce the time 364 step of the solution. A combination of DOF reduction, optimal structural damping, new gravity 365 load application, and time step reduction, was used to analyze our RC building using SC-

366 HSwithin the UI-SimCor framework, ultimately ending up with 470 effective DOFs, and a time

367 step of 0.002 . As is typical in RC structures, 5\% damping was used to form the Rayleigh 368 damping coefficients. For the large-scale HS, this damping ratio was ultimately used in the first 369 lateral and vertical modes of vibration, similar to the damping used by Kim et al. (2011). The 
370 vertical mode of vibration was kept at 5\% damping due to the fact that the final HS could result

371 in column failures and subsequent downward vertical acceleration of a line of column nodes. To

372 maintain reasonable results after such a failure, it was important not to have excessively high

373 damping in such a case.

374 Summary

375 Ultimately, although moderate success was achieved in running a SC-HS of the representative 376 building structure, a different approach was eventuallytaken to conduct a HS without many of

377 the issues associated with SC-HS, and will be described in the following section. Although these 378 solution strategies were not ultimately used for this HS, they are presented here due to their ease 379 of use and potential usefulness for other HS with complex analytical models. The solution 380 presented in the following section is much more labor-intensive and may not be appropriate or 381 feasible for other projects. The strategies presented in this section are applicable directly within 382 the current implementation of UI-SimCor, and do not require the reprogramming of 383 communication that will be discussed in the next section. However, given a model of sufficient 384 complexity (such as that used in this HS), the combination of existing techniques (numerical 385 damping, time step reduction) and newly explored strategies (gravity application procedure) 386 cannot completely resolve the issues related to SC-HS.

\section{Dynamic Hybrid Simulation (D-HS) within UI-SimCor}

Interface element between OpenSees and UI-SimCor

The methods above inherently either force sacrifices in terms of model complexity and 390 accuracy, or they have limited effectiveness and cannot completely resolve the described issues. 
391 A systematic approach to resolving the issues described above is to eliminate the constraint 392 problem from the HS altogether. Instead of performing a SC-HS, with a central coordinator 393 performing the analysis, the dynamic time integration can be performed directly within the 394 analytical substructure (OpenSees in this case), with the other "modules" treated simply as 395 experimental elements in the OpenSees structural system. Similar to OpenFresco, this type of 396 framework eliminates many of the issues that arise in SC-HS by solving the largest substructure 397 dynamically as opposed to statically, and will from now on be referred to as Dynamic Hybrid 398 Simulation (D-HS). Since the other substructures behave as experimental elements in OpenSees, 399 the tasks performed by the coordination software is reduced to communication:sending 400 displacements to physical specimens and reporting back their restoring force to OpenSees. The 401 benefit of D-HSover SC-HSis that there is no longer a large static constraint problem to be 402 solved. Note that in special cases where multiple substructures are analytical and cannot be 403 included in one model, one of them would still have to solve a set of static displacement 404 constraints to assemble the restoring force vector. The flow of communication in a D-HS is 405 similar to the procedures used in a HS using OpenFresco as a coordinator for OpenSees. This 406 project uses a D-HSarchitecture that works with UI-SimCor such that the other functions of the 407 UI-SimCor framework could be taken advantage of, such as its user interface, DAQ systems, 408 camera control, etc.Within this architecture, the dynamic time integration is performed in 409 OpenSees as opposed to the coordinator. Specific elements are defined in the model that each 410 communicate with one of the column substructures. These elements, which have been developed 411 by Dr. Oh-Sung Kwon's research group at the University of Toronto, act as interfaces to UI412 SimCor, and in OpenSees have an input stiffness matrix which remains constant throughout the 413 analysis. A diagram of the new D-HS communication procedure is shown in Figure 5. 
415 When using the standard OpenSees executable (v2.4.4), elements are dealt with in series, 416 meaning that OpenSeeswill send an element its end displacements and wait to receive restoring

417 forces before moving on to the next element in the model. This is problematic for a HS with 418 multiple substructured elements, because with this limitation of series communication, each 419 experimental column would have to wait for the previous physical specimen to finish executing 420 before continuing. Since the physical application of displacements and measurement of loads 421 typically constitutes the largest portion of time during an analysis step, this limitation can 422 effectively triple the length of a HS if there are three experimental elements, as in our study. 423 Additionally, the existing programming of UI-SimCor dictates that it communicates with all 424 modules in parallel. This contradiction between OpenSees' operation and SimCor's operation 425 causes the programs to hang up when an analysis begins. One systematic way of improving 426 communication between substructures would be to implement a continuous communication 427 similar to those implemented in OpenFresco by Shellenberg et al. (2008).

An effective way to resolve this issue was to use OpenSeesMP, which allows for multi-

429 processor applications, and therefore parallel processing. For this project, each interface element 430 between OpenSees and UI-SimCor and its corresponding nodes are explicitly defined to exist on 431 separate processors, with the remainder of the building structure located on its own processor as 432 well. This allows each experimental substructure to be dealt with in parallel, and for all 433 displacements to be sent to SimCor concurrently, allowing communication to and from the 434 coordinator as normal. Working with OpenSeesMP requires various other considerations 435 regarding recorders, solution algorithms, and other specialized commands, but those are not 436 discussed in this study (see McKenna and Fenves, 2007). 


\section{Results of GM Analysis of Full-Scale RC Frame using D-HS}

438 Using the new solution procedure described above, in which the dynamic time integration is 439 performed within OpenSees, a full-scale HS is conducted. The structural system consists of the

440 representative building described earlier, broken into 4 separate subsystems: two first story

441 columns (B1 and A1 in the building plan shown in Figure 1), one $2^{\text {nd }}$ story column (column line

442 B1 in Figure 1), and the remainder of the structure. The three single-column subsystems are

443 represented by physical specimens at the MUST-SIM NEES facility at UIUC, while the $4^{\text {th }}$ 444 subsystem, the remainder of the building, is modeled within OpenSees as described previously.

445 The structural model in OpenSees is modeled as half of the structure, taking advantage of the 446 symmetry of the building and the excitation loading (as described earlier).

447 The three physical column specimens, located at the MUST-SIM facility at UIUC, were post448 tensioned to the strongfloor at their base and to a Load and Boundary Condition Box (LBCB) at 449 their top. The LBCBs in the MUST-SIM lab each consist of a loading plate and 6 actuators, 450 oriented such that they are capable of imposing displacements in all 6 DOFs to the specimens. A 451 diagram of the specimen is shown in Figure 6a, and one of the full specimens at the MUST-SIM 452 lab is shown in Figure 6b, connected to an LBCB.

\section{3}

\section{Model Adjustment based on Column Stiffness}

Perturbations of each of the physical columns in all 6 DOFs were used to develop stiffness matrices of those elements, which are used in the OpenSees model to represent the physical columns in the stiffness of the system. Additionally, the information from these stiffness matrices is used to adjust the parameters in the analytical model such that there is consistency across physical and analytical portions of the structural frame. Since the analytical models used 
459 in OpenSees may not have the same vertical and lateral stiffness of the columns tested in the lab,

460 adjustments can be made to the model such that the load distribution among the analytical and

461 experimental columns remain as realistic as possible. For instance, if the test columns were more

462 flexible in the lateral direction than the remainder of the analytical columns on the same story,

463 the physical column would only take a small percentage of the story shear at a given story

464 drift.For this project, the constitutive relationship for the concrete model used in the fiber cross-

465 sections is adjusted (specifically the strain at peaks stress) such that lateral stiffness of

466 experimental columns and analytical columns are consistent. As will be shown in the following

467 section, lateral stiffness matching is more important than vertical stiffness matching because

468 relatively large discrepancies in vertical stiffness result in small changes in gravity load

469 distribution, such that matching vertical stiffness is not imperative in this study.

470 Gravity load application results

471 The gravity loads in the HS are applied such that the loads are increased incrementally, with

472 a static hold period after the total load is applied, allowing the structure to slowly resolve the

473 unbalanced force and reach equilibrium (while maintaining compatibility). To show the

474 effectiveness of this procedure, the axial forces on columns B1 and B2 (one physical column and

475 one analytical column) are shown from the hybrid analysis in Figure 7, with dashed horizontal

476 lines representing the gravity loads that those same columns hold in a purely analytical solution

477 from OpenSees. The loads developed in the columns are shown to reach their expected values.

478 Note that these loads are not imposed directly to these members, but rather incrementally solved

479 using structural analysis (through applied displacements),with the internal gravity forces

480 developing as a result of those displacements (a system-level load application using OpenSees). 
Figure 8 shows the vertical displacement at the top of one of those columns (experimental

482 column B1 on the first story). Comparing purely analytical results with the HS shows that the 483 experimental column needed to deform downwards approximately an additional $0.28 \mathrm{~mm}\left(0.011^{\prime \prime}\right)$, 484 or in other words an additional $25 \%$ displacement, to reach equilibrium with the remainder of the 485 structure. However, as shown in Figure 7, this large relative difference in displacement only 486 corresponds to a negligible change in the supported axial force in that column. This is due to the 487 fact that the flexural stiffness of the beams in the frame is significantly smaller than the axial 488 stiffness of the columns. In other words, although the relative change in vertical displacement $489(25 \%)$ between physical and analytical results is large, the absolute change $\left(0.28 \mathrm{~mm}, 0.011^{\prime \prime}\right)$ is 490 still far too small for a meaningful amount of shear to develop in the surrounding beams, which 491 would be required for the gravity loads to redistribute elsewhere. Again, this can be concluded 492 from the observation of axial force in B1 due to the system-level solution of the gravity loading. 493 In order for beams to develop additional shear or for surrounding columns to take on additional 494 axial forces, the axial force in B1 would need to drop with respect to the analytical results (which 495 it does not). The conclusion from this observation is that large changes in column vertical 496 stiffness can have a negligible effect on the gravity load distribution in the system.

\section{Earthquake GManalysis results}

498 After imposing gravity loads to the system, and to verify the HS procedure, the structure is 499 subjected to an $85 \%$ scaled GM of the east-west recording of the Lucerne station of the 1992 500 Landers, CA earthquake. The motion is imposed in the transverse direction of the structure. Due 501 to the magnitude of the motion being small early in the GM recording, analyses and simulations 502 are performed beginning 4 seconds into the GM record. The results are compared to the results 503 of a non-hybrid analysis run directly within OpenSees (consistent with the model shown in 
504 Figure 2). A comparison of the time-history of the top building displacement in the two cases is

505 shown in Figure 9.Note that the analysis was started at $4 \mathrm{~s}$ into the GM due to there being 506 minimal excitation prior to that point. The test is shown up through $13 \mathrm{~s}$, after which motions are 507 smaller and dissipate, as the main pulse occurs from $10.5 \mathrm{~s}$ to $12.5 \mathrm{~s}$ of the motion. Subsequent 508 figures also present the results during this same time period.

509 Although this HS does not lead to column failure or structural collapse, the demand on the 510 columns and the other building components is significant. Physical specimens experienced 511 significant flexural cracking, and the beams of the analytical portion of the model developed 512 plastic hinges in many locations. It is therefore important to confirm that the nonlinear behavior 513 of the HS is in agreement with the previous analytical model results to verifyour modeling and 514 analysis procedure.

515 Since it is apparent that global responses should not be affected by the inclusion of physical 516 test specimens into the analysis (since no failures occur), more localized responses are perhaps a 517 better measure of the performance and accuracy of the HS. Figure 10 shows the $1^{\text {st }}$ story shear in 518 the first two frames of the structure (Frames 1 and 2 in Figure 1). Comparing the results of the 519 hybrid test with the analytical results from OpenSees, there is less than a 5\% difference between 520 the two cases at peak displacement. This illustrates the legitimacy of the hybrid testing approach, 521 as the shear in frame 1 during the HS is a direct result of shear contributions from two of the test 522 columns and one analytical column in the building model. Figure 11 shows the shear force in 523 column B1 on the $2^{\text {nd }}$ story, one of the experimental test columns. The overall comparison of the 524 two analyses shows consistent results, with a difference at peak of less than 5\%.

525 During the main pulse of the ground motion, Column A1 on the $1^{\text {st }}$ story is subjected to 526 additional compression due to overturning moment. This additional compression, along with the 
527 orientation of the actuators in the LBCB and issues with hydraulics (actuator capacity was lower

528 than expected), caused one of the vertical actuators to hit its force capacity limit prior to the end

529 of the test. Once this limit was reached, LBCB2 was unable to accurately impose the

530 commanded displacements from the OpenSees model onto the test specimen and return the

531 forces to UI-SimCor. Typically problems such as this can abruptly end a HS, as the physical

532 elements in the lab are unable to move forward. However, during this HS a solution was found

533 that allowed the test to proceed using the remaining test specimens.

\section{I-modification and its use for mitigating actuator capacity limits}

535 When introducing physical components to a model and the addition of the associated

536 experimental error, stability issues can arise in HS. Due to limitations in actuator displacement

537 accuracy, deformations within the test structure, and resolution of internal measurement sensors,

538 a physical component within a hybrid analysis may not be subjected to its exact prescribed

539 displacement. This error in displacement obviously results in a likewise error in force reported

540 back to the analytical model. More detailed discussion of this error and its effects can be found in

541 Combescure and Pegon (1997). To combat this limitation of physical control, "I-modification" is

542 used. I-modification is a technique used to correct resulting forces from a physical specimen by

543 multiplying the displacement error (calculated by using higher-precision external measurement

544 sensors) by the stiffness matrix of the physical element, and subtracting that theoretical force

545 error from the measured force value. Typically the initial (maximum) stiffness of the physical

546 element is used, which has the tendency to artificially damp out force errors (Combescure and

547 Pegon, 1997). Note that if there is a large change in element stiffness and/or strength during a

548 test, one must be careful to keep experimental movement errors small, or the I-modification will

549 erroneously introduce an excessively large correction to the resulting forces. Therefore, after 
550 element yielding, damage, or total failure, error tolerances must be tightening in the laboratory 551 software.

552 Typically I-modification is used as a stabilization term, correcting the force response for small, 553 unavoidable, but measurable errors in physical specimen displacements caused by the limitations 554 of actuator accuracy and precision. However, even when displacement errors are large, provided 555 that the system remains relatively linear, these corrections from I-modification can still be 556 effective in mitigating the potential discrepancies in force response. In this experiment, since 557 there was effectively a loss of control of LBCB2 when one of the actuators hit a capacity 558 limit,the I-modification caused any incremental displacements that were prescribed after the loss 559 of control of LBCB2 to effectively be treated as displacements applied to a linear element, and 560 added this term to the actual force response of the column (under the incorrect displacement). 561 The vertical response of Column A1 on the first story, with and without the I-modification 562 corrections, are shown in Figure 12, and are compared with the computational desktop analysis 563 results. It can be seen that the I-modification effectively mitigates the actuator strength limit, 564 adjusting the restoring force sent back to the model to a reasonable value. This procedure uses 565 every piece of experimental data available after losing actuator control, by only analytically 566 formulating the portion of the force resulting from the displacement error (using I-modification).

567 Although this actuator capacity limit and subsequent corrections to the restoring force sent 568 back to the analytical model dictate that detailed discussion and analysis of that column beyond 569 this point of the test would be misleading, the resulting response generated from the I570 modification corrections show good agreement with the nonlinear OpenSeesanalytical results. 571 This agreement indicates performing these corrections allows for successful continuation of 
572 testing on the remaining components of the HS. This allows the HS to continue and observations

573 on the remaining two physical specimens to be made.

\section{Overall agreement of $H S$ results with OpenSees data}

575 As shown in Figures 7 through 12, the D-HS using UI-SimCor with OpenSees shows good 576 agreement with a purely analytical OpenSees model, both at the global level, such as top 577 building displacement, as well as at a more local level, such as the column axial and shear forces.

578 Figure 13 also shows the $1^{\text {st }}$ story drift, which perhaps is most affected by the experimental 579 specimens, as they account for a large percentage of the story shear on the $1^{\text {st }}$ story. Again, there 580 is good agreement between the D-HS results and the purely analytical OpenSees results.

\section{Conclusions}

582 A large-scale hybrid simulation is conducted at the MUST-SIM NEES facility at the University 583 of Illinois at Urbana Champaign (UIUC), using the modified UI-SimCor simulation tool and 584 architecture. The complexity of the analytical model required by the objective of this HS was 585 beyond that which has been done in the past using UI-SimCor, which is shown to introduce 586 several difficulties. Multiple mitigation methods are proposed in order to conduct a full-scale 587 hybrid simulation of a 10-story RC structure.Note that the results of a second HS in which 588 experimental columns fail is beyond the scope of this paper and are not discussed here.

589 When performing a statically-condensed hybrid simulation (SC-HS), where there is a need

590 for static analysis of an analytical substructure, a large and potentially demanding constraint 591 problem will be created if the number of effectiveDOFs becomes large within the analytical 592 substructure. When the analytical substructure is modeled nonlinearly, this constraint problem 593 can lead to convergence issues in implicit integration of the analytical model. Additionally, it is 
594 shown that the alpha-OS method causes large oscillations in the higher-frequency modes of the 595 HS model used in this project. It is shown that for this study, significant reduction of the 596 effective DOFs along with reduction of the time step size, optimal use of numerical alpha 597 damping and high amounts of viscous damping in higher modes are all needed toreduce the 598 convergence and oscillation issues related to the use of a complex analytical substructure in a 599 SC-HS.

600 A new method of gravity load application for implementation in SC-HS is proposed, in 601 which gravity loads are pre-applied directly within a substructure(s). This procedure effectively 602 condenses out a large portion of the degrees of freedom analyzed dynamically by the 603 coordination software.

604 Due to the issues caused in the SC-HS of the 10-story RC building model, and the associated 605 need for reduction of model complexity to obtain reasonable results, a different approach is taken 606 to conduct a D-HS, in which the dynamic time-integration is performed within OpenSees. This 607 approach allows for increased model complexity without having issues with large oscillations or 608 having a large potential for convergence issues due to static solution of a large constraint 609 problem.

610 The multiprocessor capabilities of OpenSeesMP are shown to be effective in significantly 611 reducing the duration of a hybrid simulation in which multiple experimental substuctures are 612 needed. Lastly, although loss of experimental control of a specimen (due to actuator limitations) 613 introduces varying levels of error and uncertainty, it is shown that the I-modification technique 614 described by Combescure (1997) can be used to continue structural testing well beyond the limits 615 (force or displacement) of the actuators of a particular specimen, enabling a test to continue with 616 the remaining physical specimens. 
617 Using the assortment of changes and adjustments to UI-SimCor and the hybrid simulation 618 procedure at the MUST-SIM facility, a large-scale HS is conducted using a complex analytical

619 model. The results of the analysis are consistent with the results of a desktop simulation. Since

620 the $85 \%$ scaled GM did not produce any failures, the HS are expected to show agreement with 621 analytical results. This test represents a complete system-level response analysis, as the model 622 consists of a 3D RC frame modeling of a 10-story building. The model which was ultimately 623 used in the hybrid simulation has a complexity more than an order of magnitude larger than the 624 previous hybrid tests using similar architecture, representing a step toward more flexibility in the 625 use of hybrid simulations in the future.

\section{Acknowledgements}

627 This paper is based upon research supported by the National Science Foundation under Grant 628 No. NEES/CMMI-1135005. The authors greatly appreciate this support. A special thanks to Dr. 629 Oh-Sung Kwon for his help with creating an interface element for communication between 630 OpenSees and UI-SimCor. His help is greatly appreciated. The authors would also like to thank 631 Dr. Bill Spencer andDr. Dan Kuchma, as well as the rest of the team at UIUC for their help 632 during the experiment, including Michael Bletzinger, Mike Johnson, Tim Prunkard, Wes Walton, 633 Anahid Behrouzi, Dr. DoSoo Moon, and Dr. Chia-Ming Chang.

\section{References}

635 Abdelnaby A., Frankie T.,Spencer B., (2014). "Numerical and hybrid analysis of a curved bridge 636 and methods of numerical model calibration." Engineering Structures 70C, 234-245.

637 ACI 318-63, 1963. Building Code Requirement for Reinforced Concrete, American Concrete 
Institute, MI.

639 Chopra A.K., 2012. Dynamics of Structures: Theory and Applications to Earthquake

640 Engineering, 4th Edition, Prentice Hall, Englewood Cliffs, NJ.

641 Combescure D. and Pegon P., 1997. "alpha-Operator Splitting time integration technique for pseudodynamic testing - Error propagation analysis, ” Soil Dynamics and Earthquake Engineering, 16(7-8), 427-443.

644 Del Carpio, M., Mosqueda, G. and Hashemi, M.J., 2015. "Large-Scale Hybrid Simulation of a Steel Moment Frame Building Structure through Collapse."Journal of Structural Engineering, 10.1061/(ASCE)ST.1943-541X.0001328Elnashai, A.S., Spencer, B.F., Kuchma, D., Ghaboussi, J., Hashash, Y. and Quan, G.,2004. "Multi-AxialFull-Scale SubStructured Testing and Simulation (MUST-SIM) facility at the University of Illinois

Elwood, K.J., 2004. "Modelling failures in existing reinforced concrete columns," Canadian atUrbana-Champaign,"Proceedings of the 13th World Conference on Earthquake Journal of Civil Engineering, 31(5), 846-859.

GSA, 2003. "Progressive Collapse Analysis and Design Guidelines for New Federal Office Buildings and Major Modernization Projects," U.S. General Service Administration, 656 Henkhaus, K., Pujol, S., and Ramirez, J. 2013. ”Axial Failure of Reinforced Concrete Columns Washington, DC. 
by JohnA. Blume \& Associates, San Fernando, California Earthquake of February 9, 1971,” U.S. Departmentof Commerce, National Oceanic and Atmospheric Administration, 1(A), 301-443.

Kim S.J., Holub C.J., and Elnashai A.S., 2011."Experimental investigation of the behavior of RC bridge piers subjected to horizontal and vertical earthquake motion,"Engineering

Kwon, O.S., Elnashai, A.S., and Spencer, B.F., 2008. "A framework for distributed analytical and hybrid simulations," Structural Engineering and Mechanics, 30(3), 331-350.

Kwon O.S., Nakata N., Elnashai A.S., and Spencer B., 2005. “A Framework for Multi-Site Structures, 33(7), July 2011, 2221-2235.

\section{1}

672

673

674

Distributed Simulation and Application to Complex Structural Systems,"Journal of Earthquake Engineering, 9(5), 741-753.

Kwon O.S., Nakata N., Park K.S., Elnashai A., and Spencer B., 2007. "User Manual and Examples for UI-SIMCOR v2.6," University of Illinois at Urbana-Champaign, IL.

LinS., 2010.“An integrated earthquake impact assessment system,” Ph.D. dissertations, Department of Civil and Environmental Engineering,University of Illinois, Urbana, IL.

Li, J., B. Spencer, and Elnashai, A. (2013). "Bayesian Updating of Fragility Functions using Hybrid Simulation." Journal of Structural Engineering 139(7): 1134-1148.

Lynn, A. C., Moehle, J. P., Mahon, S. A., and William, W. T., 1996. "Seismic evaluation of existing reinforced concrete building columns," Earthquake Spectra, 12(4), 715-739.

MacLeod, I. A., 1990. “Analytical Modeling of Structural Systems-An Entirely New Approach with Emphasis on Behavior of Building Structures," Ellis Horwood Series in Civil Engineering, UK. 
682 Mahmoud, H. and Elnashai, A., 2013. "Hybrid Simulation of Semi-rigid Partial-Strength Steel 683 Frames," Structures Congress 2013, 2410-2420.

684 McKenna F. and Fenves G., 2007. "Using the OpenSees Interpreter on Parallel Computers," 685 NEESit: Enabling the Network for Earthquake Engineering Simulation TN-2007-16.

686 McKenna F., Fenves G.L., and Scott M., 2002. Open system for earthquake engineering 687 simulation (OpenSees), University of California, Berkeley.

688 Murray, J.A. and Sasani, M., 2013.“Seismic shear-axial failure of reinforced concrete columnsvs. 689 system level structural collapse,"Engineering Failure Analysis, 32, September 2013, 382$690 \quad 401$.

691 Murray J.A. and Sasani M., 2014."Evaluating System-Level Collapse Resistance of Non-Ductile RC Frames Structures," Proceedings of the 10th National Conference in Earthquake Engineering, Earthquake Engineering ResearchInstitute, Anchorage, AK.

694 Nakashima M. and Kato H., 1988. Experimental Error Growth Behavior and Error Growth 695 Control in Pseudo Dynamic Test. Proceedings of Ninth World Conference on Earthquake 696 Engineering. Tokyo-Kyoto, Japan.

697 Sasani, M., 2007. "Life-safety and near-collapse capacity models for seismic shear behavior of 698 RCcolumns," Structural Journal, ACI, 104(1), 30-38.

699 Sasani, M., Kazemi, A., Sagiroglu, S., and Forest, S., 2011. "Progressive collapse resistance of 700 an actual 11-story structure subjected to severe initial damage," Journal of Structural Engineering, ASCE, 137(9), 893-902.

702 Schellenberg, A. H., Huang, Y., and Mahin, S. A., 2008. "Structural FE-software coupling 703 through the experimental software framework, OpenFresco." Proceedings of the 14th 
World Conf. Earthquake Engineering, Beijing.

705 Schellenberg A., Kim H., Patterson D., Mahin S.A.,2010. "OpenFresco," University of 706 California Berkeley, CA. https://nees.org/resources/OpenFresco.

707 Schellenberg A., Mahin S.A., Fenves G.L., 2007.“A software framework for hybridsimulation of 708 large structural systems,"Proceedings of the structural engineering research frontiers, Long $709 \quad$ Beach, California, USA.

710 Sezen, H., 2002. "Seismic behavior and modeling of reinforced concrete building columns,"

711 Ph.D. Dissertation, Department of Civil and Environmental Engineering, University of 712 California, CA.

713 Shao, X. and Griffith, C., 2013.“An overview of hybrid simulation implementations in NEES 714 projects”, Engineering Structures, 56, November 2013, 1439-1451.

715 Shing, P. B., Stavridis, A., Wei, Z., Stauffer, E., Wallen, R., and Jung, R.-Y., 2007. "Validation 716 of a fasthybrid test system with substructure tests," 17th Analysis and Computation 717 Specialty Conference, St.Louis, MO.

718 Spencer Jr., B. F., Elnashai, A., Kuchma, D., Kim, S., Holub, C., and Nakata, N., 2006. "Multi719 site Soil-Structure-Foundation Interaction Test (MISST)," University of Illinois at Urbana720 Champaign.

721 Takanashi, K., Udagawa, K., Seki, M., Okada, T., and Tanaka, H., 1975. "Nonlinear earthquake 722 response analysis of structures by a computer-actuator on-line system," Bulletin of 723 Earthquake Resistant Structure Research Centre No.8 Institute of Industrial Science, $724 \quad$ University of Tokyo, Japan.

725 Yang T.Y., Stojadinovic B., Moehle J., 2009.“Hybrid simulation of a zipper-bracedsteel frame 


\section{$727 \quad$ List of Figures}

728 Figure 1. Typical floor plan of representative RC structure

729 Figure 2. Typical node and element layout in floor plan of OpenSees RC frame model

730 Figure 3. Flowchart of SC-HS procedure using UI-SimCor

731 Figure 4. Column 10300 axial force vs. step number

732 Figure 5. New communication loop for dynamically-analyzed hybrid simulation (D-HS)

733 Figure 6. (a) Technical drawing of test specimen (b) photo of specimen at MUST-SIM lab

734 Figure 7. Axial forces in 1st story columns B1 (experimental) and B2 (analytical), with gravity

735 loads from purely analytical results shown as dotted lines

736 Figure 8. Vertical displacement at the top of column B1 on the 1st story

737 Figure 9. Top building displacement of hybrid simulation compared with OpenSees model

738 Figure 10. Frame shear comparisons on 1st story

739 Figure 11. Shear force in 2nd story column B1

740 Figure 12. Axial force in Column A1 on the 1st story with and without I-modification, compared

741 with desktop analysis results

742 Figure 13. First story drift of hybrid simulation compared with OpenSees model 

(1)
(2)
(3)
(4)
(5)
(6)
(7)

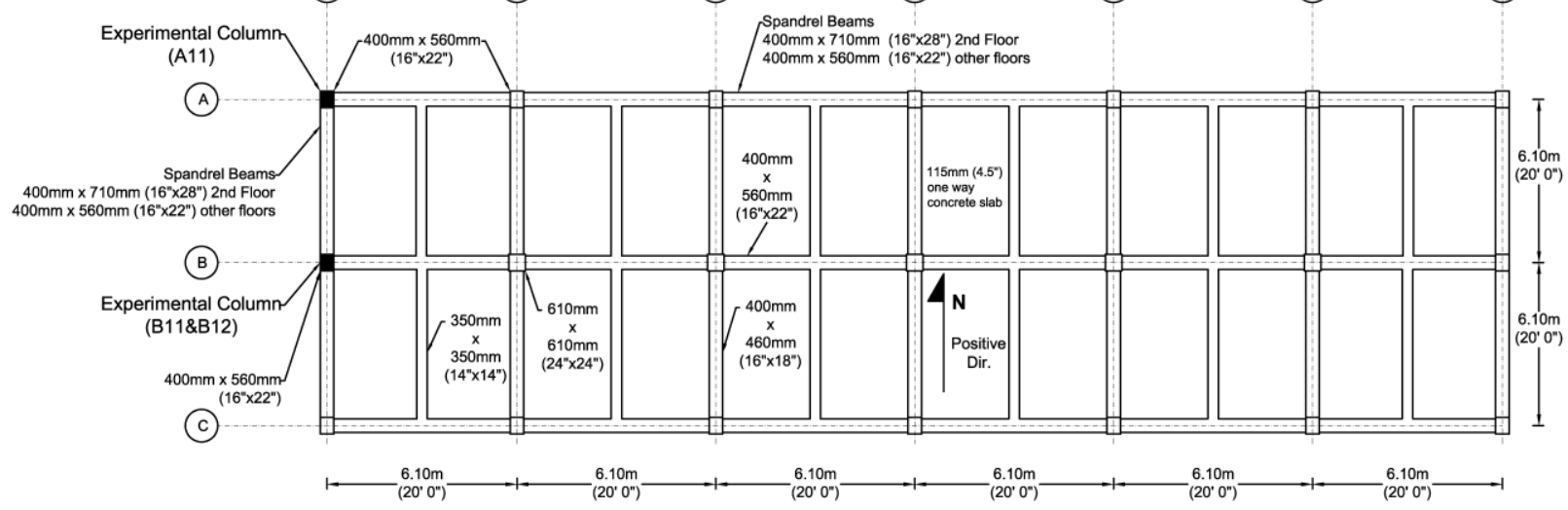


(1)

(2)

(3)

(4)

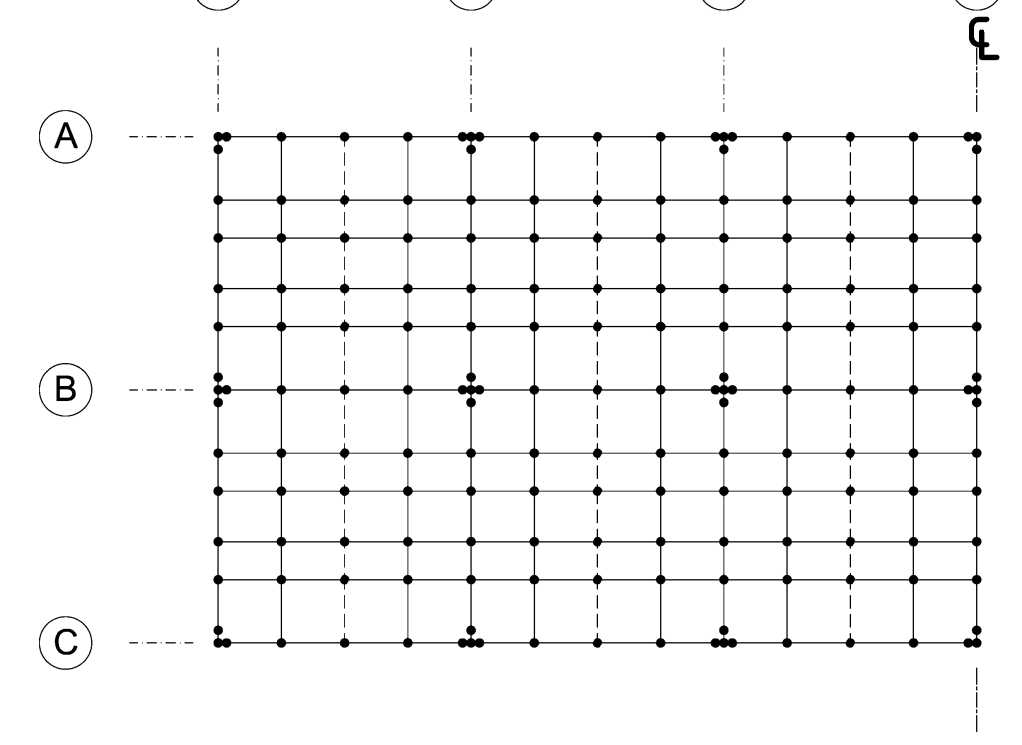

746 Figure 2. Typical node and element layout in floor plan of OpenSees RC frame model 

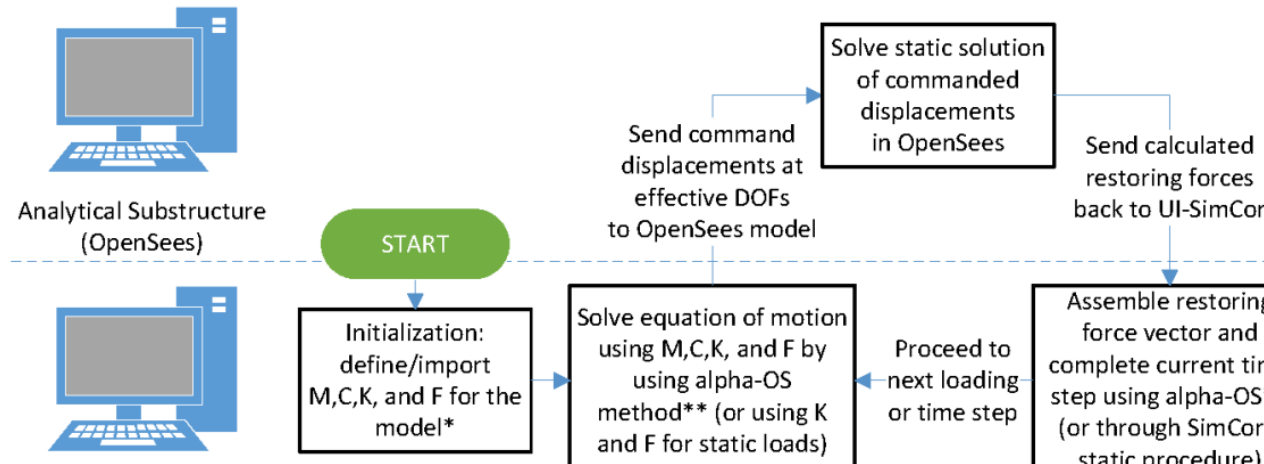
back to UI-SimCor

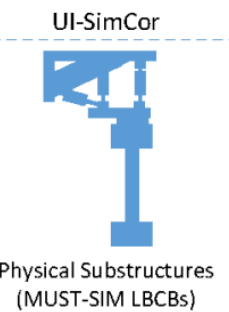

* M, C, K, and F are only defined at the "Effective Degrees of Freedom", essentially condensing out any DOFs which have no mass or loads

** More detailed description of the alpha-OS method can be found in Combescure and Pegon (1997)

Figure3. Flowchart of SC-HSprocedure using UI-SimCor 


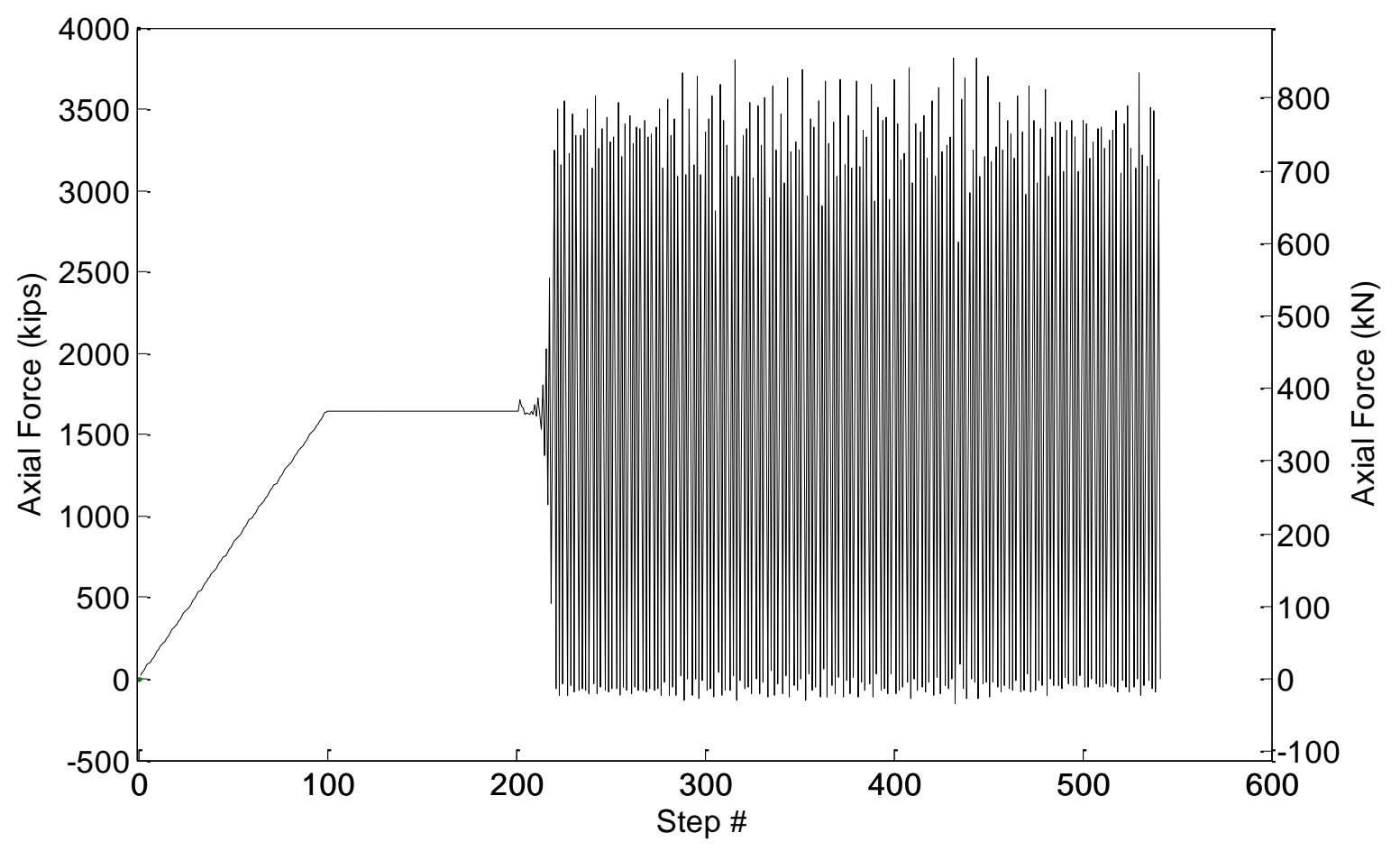

749

750

Figure4.Axial force vs. step number of column B1 on the $1^{\text {st }}$ story 


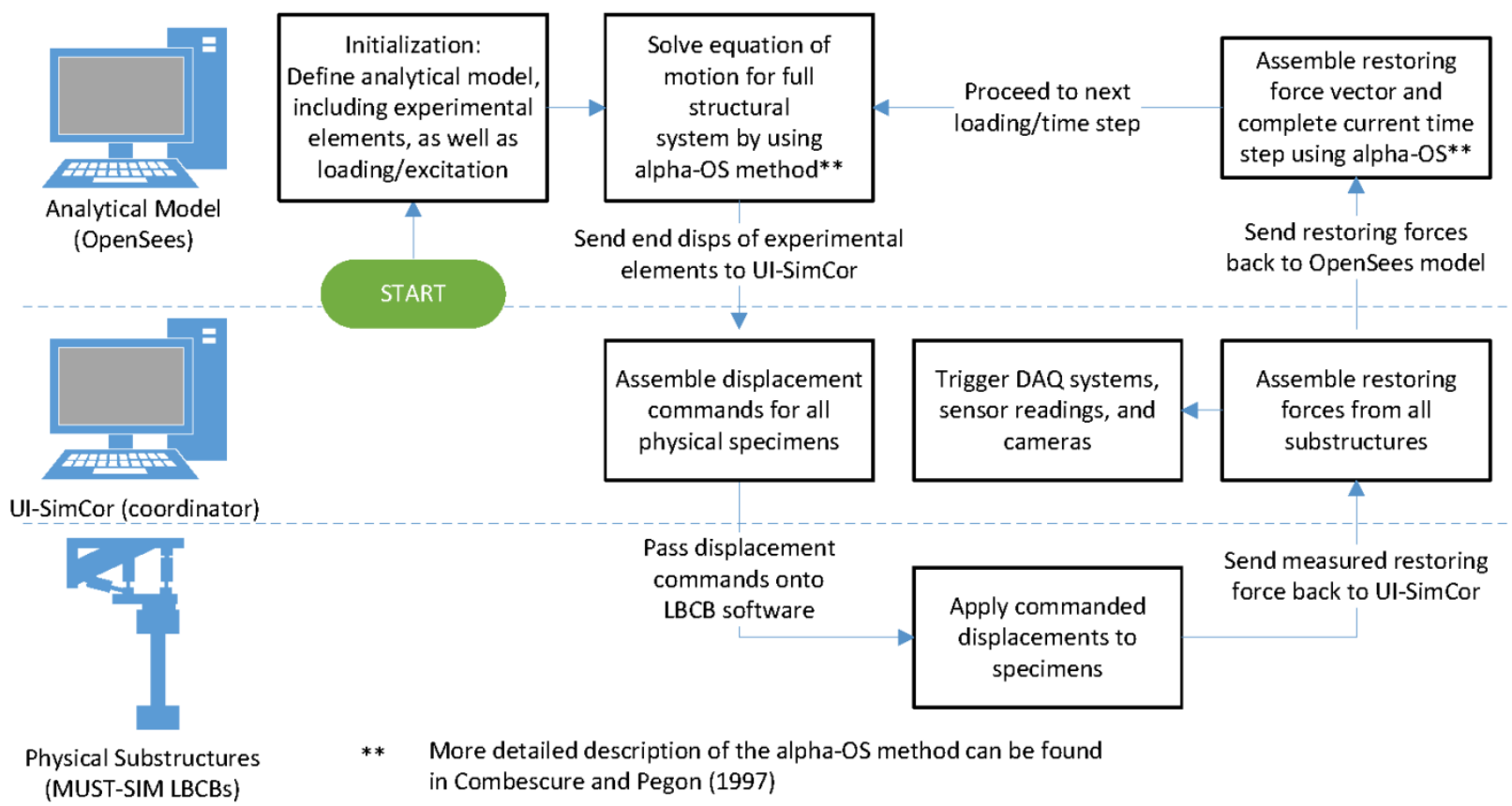

752 Figure5. New communication loop for dynamically-analyzed hybrid simulation (D-HS) 

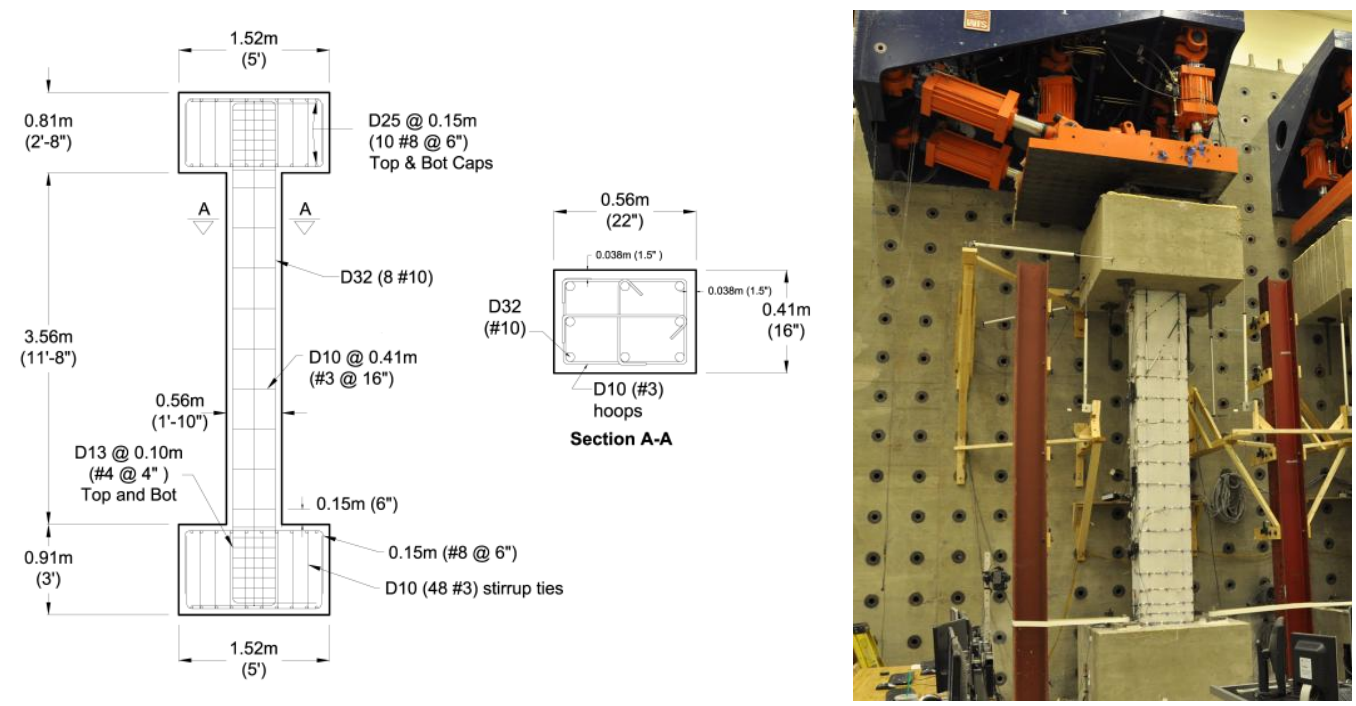

754 Figure 6. (a) Technical drawing of test specimen (b) photo of specimen at MUST-SIM lab 


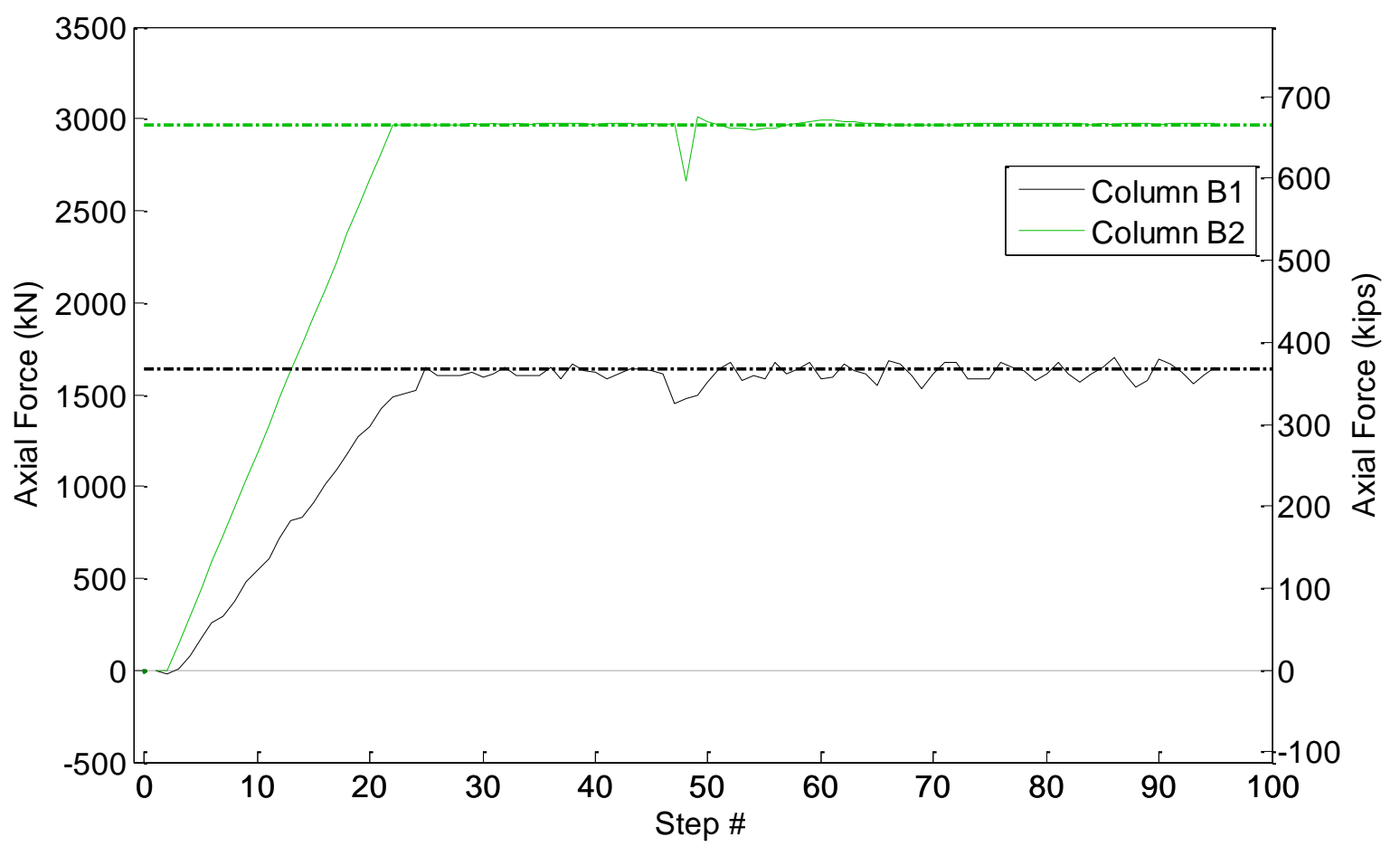

757 Figure 7. Axial forces in $1^{\text {st }}$ story columns B1 (experimental) and B2 (analytical), with gravity 758 loads from purely analytical results shown as dotted lines 


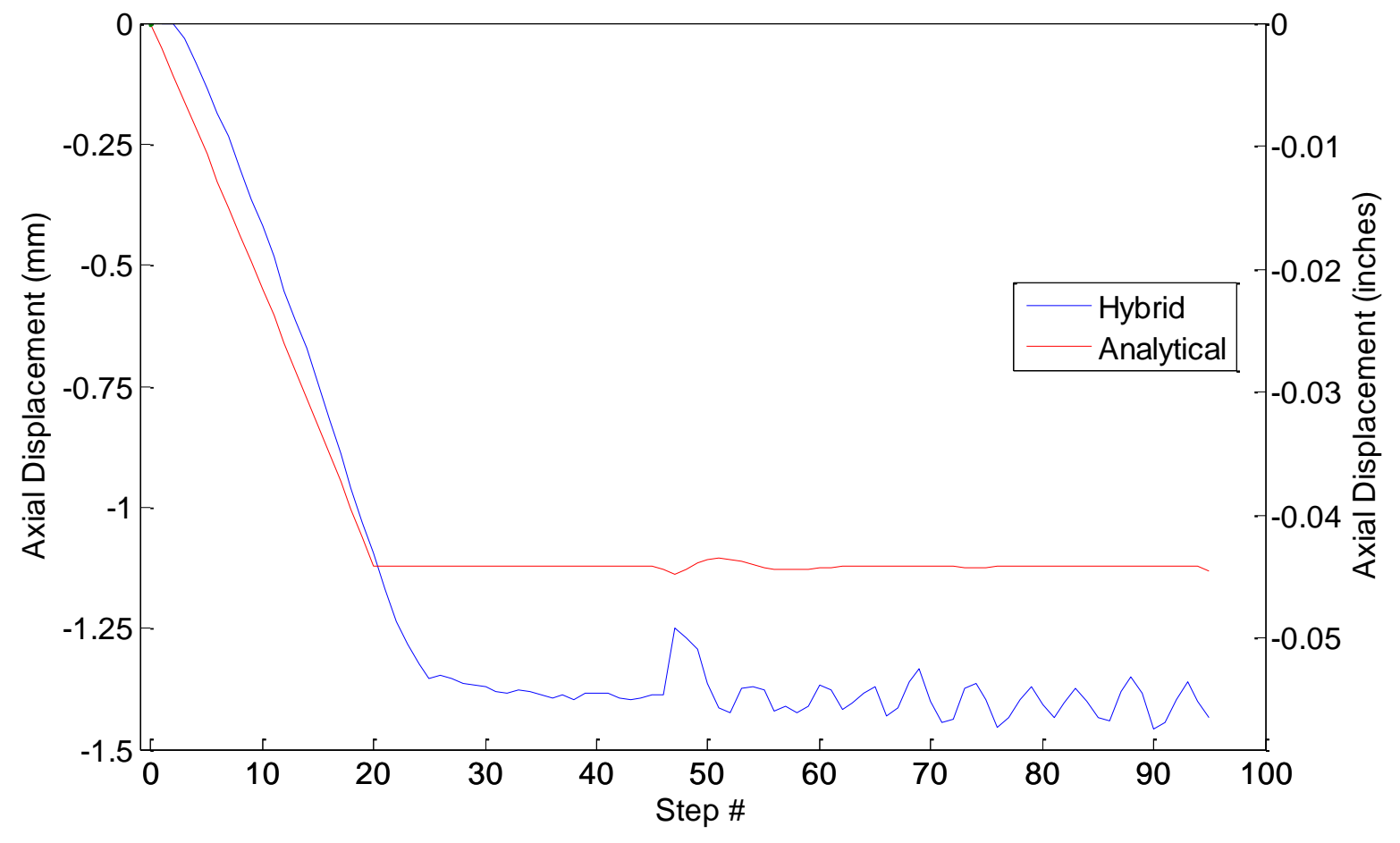

759

Figure 8 . Vertical displacement at the top of column B1 on the $1^{\text {st }}$ story 


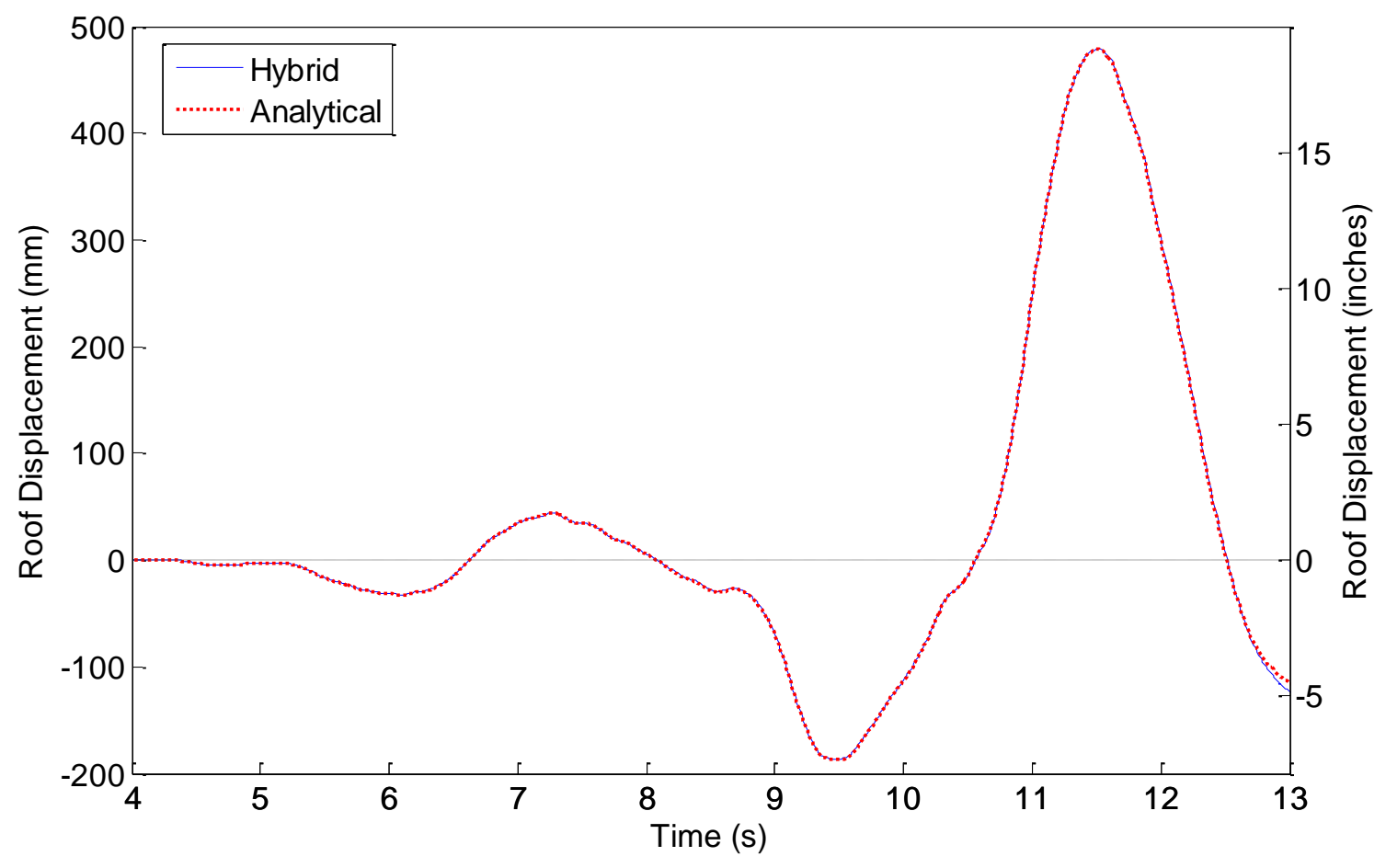

762 Figure 9. Top building displacement of hybrid simulation compared with OpenSeesmodel 


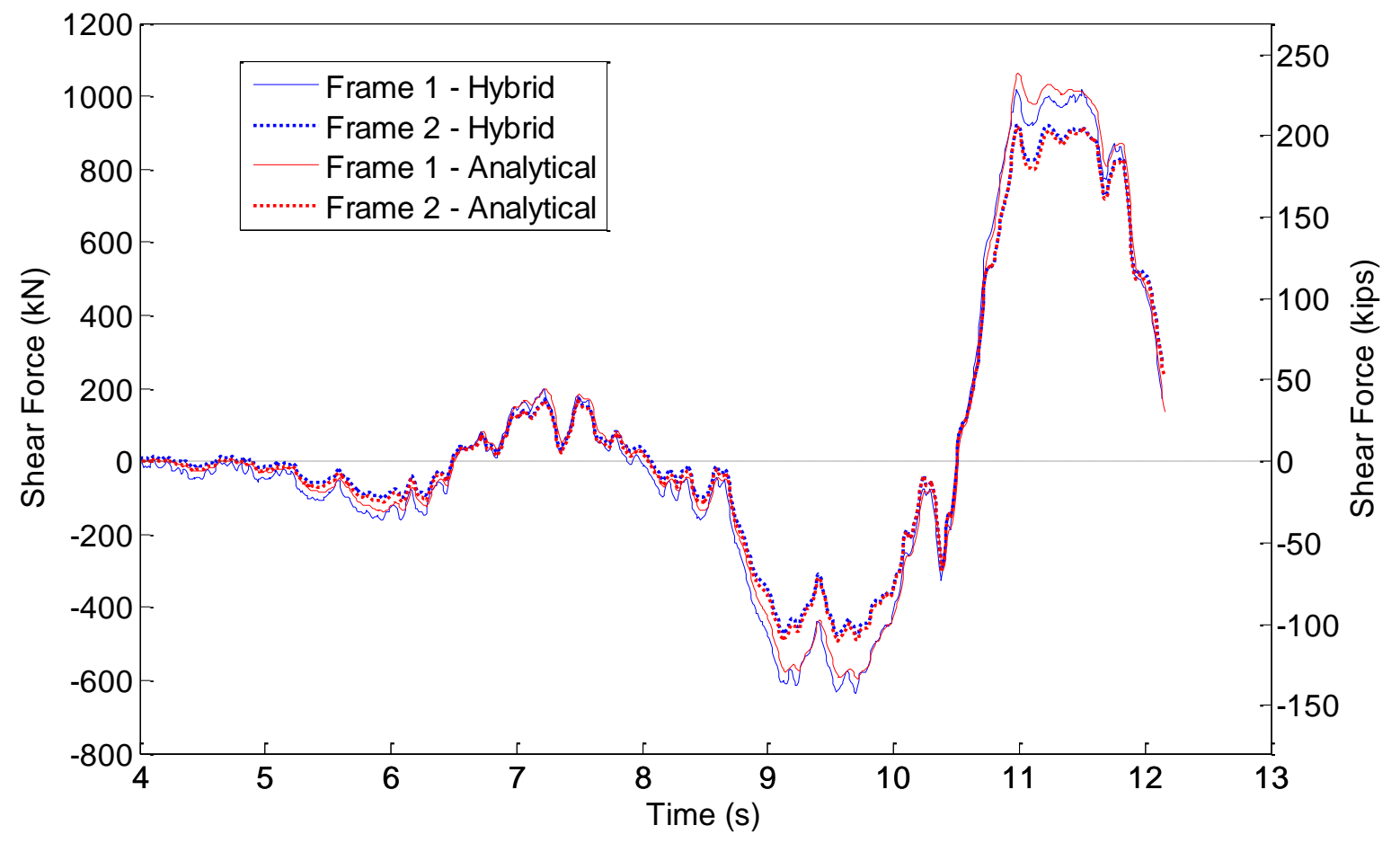

Figure 10. Frame shear comparisons on $1^{\text {st }}$ story 


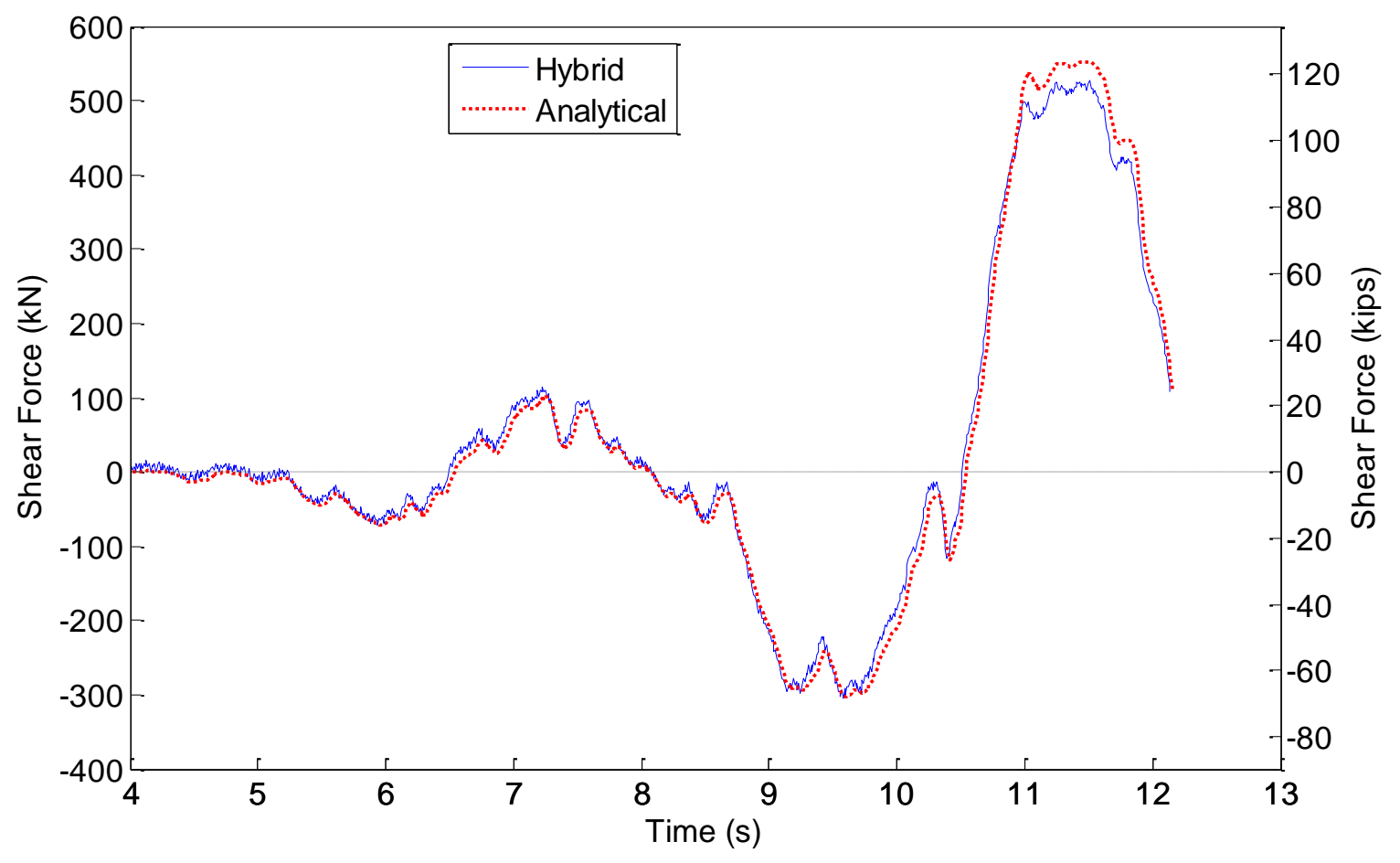

Figure 11. Shear force in $2^{\text {nd }}$ story column B1 


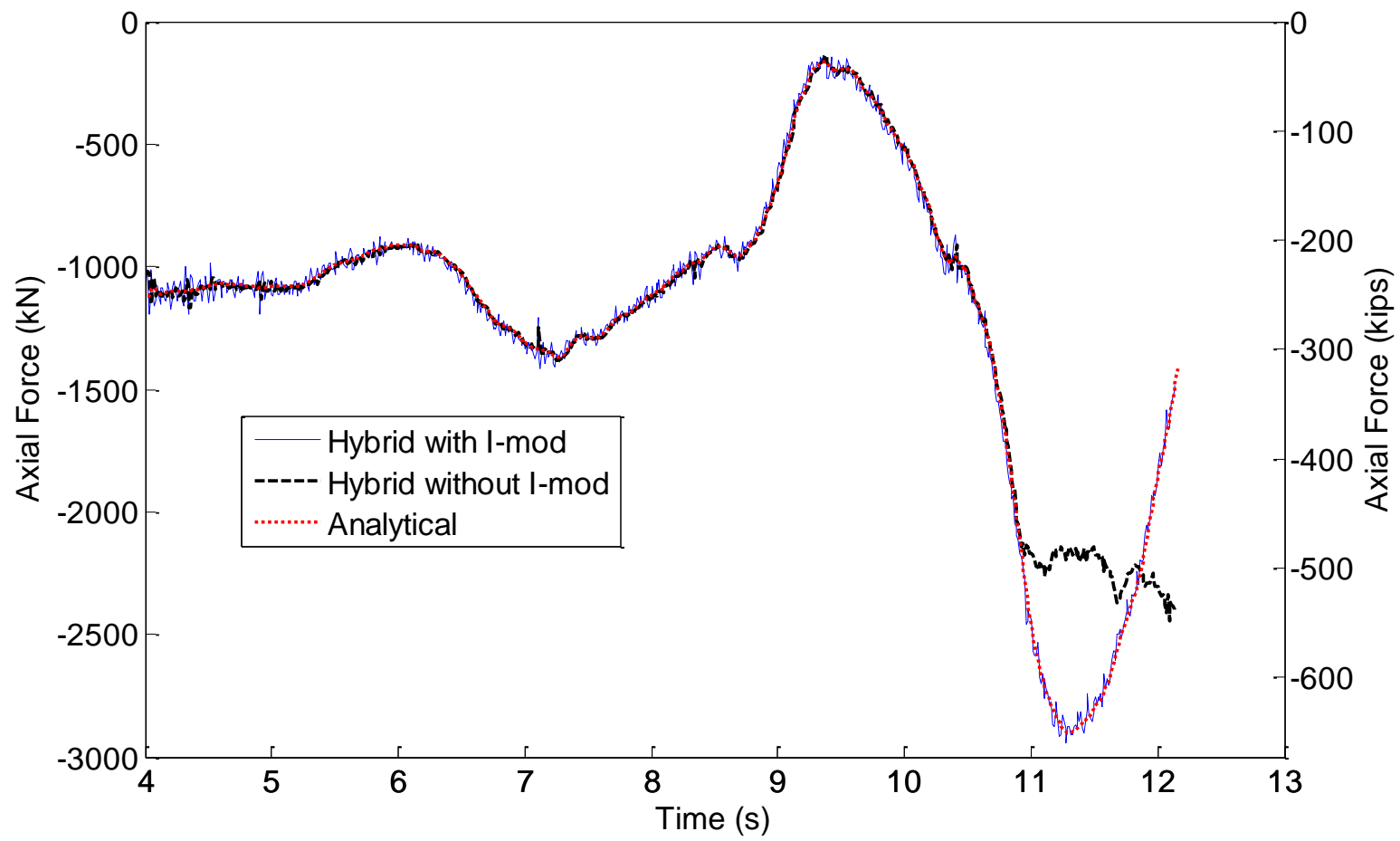

Figure 12. Axial force in Column A1 on the $1^{\text {st }}$ story with and without I-modification, compared with desktop analysis results 


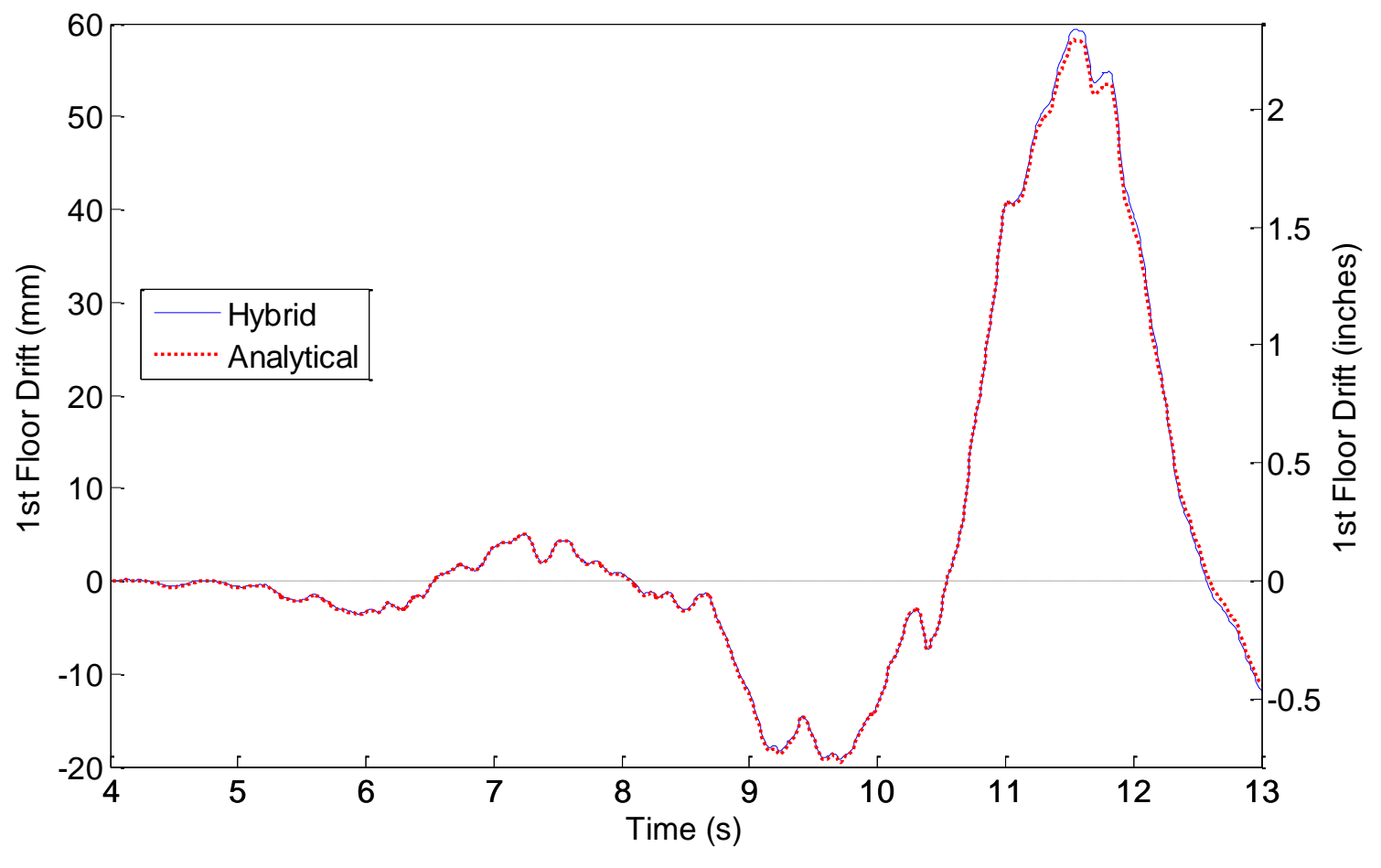

771

772

Figure 13. First story drift of hybrid simulation compared with OpenSeesmodel 